\title{
Subdifferential calculus for invariant linear ordered vector space-valued operators and applications
}

\author{
Antonio Boccuto \\ Dipartimento di Matematica e Informatica, University of Perugia, via Vanvitelli, 1 - I-06123 Perugia (Italy) \\ antonio.boccuto@unipg.it
}

\begin{abstract}
We give a direct proof of sandwich-type theorems for linear invariant partially ordered vector space operators in the setting of convexity. As consequences, we deduce equivalence results between sandwich, Hahn-Banach, separation and Kreintype extension theorems, Fenchel duality, Farkas and Kuhn-Tucker-type minimization results and subdifferential formulas in the context of invariance. As applications, we give Tarski-type extension theorems and related examples for vector lattice-valued invariant probabilities, defined on suitable kinds of events.
\end{abstract}

\section{Keywords}

Dedekind complete partial ordered vector space, amenability, Hahn-Banach theorem, sandwich theorem, Fenchel duality theorem, Krein extension theorem, subdifferential.

\section{Academic Discipline And Sub-Disciplines}

Mathematics

\section{MATHEMATICS SUBJECT CLASSIFICATION}

28B15, 43A07, 46N10, 47N10.

\section{TYPE (METHOD/APPROACH)}

Research article; Quasi-Axiomatic with Applications.

\section{INTRODUCTION}

Sandwich and Hahn-Banach-type theorems are deeply studied, have several applications in different areas, and are related with several topics, among which, for example, minimization of functionals and operators. In these cases, often it is advisable to associate to a "primal" problem a "dual" problem, which is in general easier to handle, and to investigate the relations existing between them. These tools are widely used, for instance, in reconstructing images corrupted by noise and/or blur (see also $[9,17]$ ), in which it is possible to minimize the primal energy by investigating the dual energy and to deal with suitable convex approximations. There is a very wide literature concerning the Fenchel duality theorem, which are often used, for example, in problems of convex analysis, numerical analysis and Calculus of Variations (see also $[1,2,37,40])$.

Another kind of problems connected with sandwich-type theorems deal with nonlinear minimization programming, in which some constraints are often posed, and there are several studies on finding related necessary and/or sufficient conditions, for example saddle-type properties. Among them, we quote in particular Farkas and Kuhn-Tucker-type theorems (for a related literature and an overview, see for instance $[19,27,30,46])$.

Other applications and consequences of Hahn-Banach theorems can be found in separation theorems on convex sets by means of an affine manifold (see also [26]), in (Riesz) MV-algebras (see also [6,20,33]), in subdifferential calculus and its various features (see also [11,12,31]). In the literature, many studies about these topics have been extended to the context of partially ordered space-valued operators and measures (see also $[8,10,14,24,32]$ and their bibliographies).

Another field of research related with sandwich-type theorems are extension theorems for finitely additive (and invariant) measures and probabilities, for example in exchangeable processes (see also [4,28,39]). Some theorems of this kind for invariant partially order vector space-operators were given, for instance, in [3,7,15,25,41,42]. In particular, in [3] and [7] some characterizations of amenable (semi)groups were given, in terms of these kinds of theorems, in the context of vector lattice-valued invariant operators and set functions.

Finally, in [6], some separation theorems by means of affine invariant manifolds were given, together with Farkas and Kuhn-Tucker-type theorems, in the context of partially ordered vector spaces.

In this paper, we first prove a sandwich-type theorem for invariant linear ordered vector space-valued operators in the setting of convexity, using a similar result proved in [47], when the involved linear operators are not necessarily invariant. Successively, some Fenchel duality-type theorems, subdifferential formulas, Hahn-Banach and Krein extension theorems 
are proved, and their equivalence with separation, Farkas and Kuhn-Tucker-type theorems are shown. Our techniques are inspired by methods used in [21,22,34,35,36,45,46,47]. Furthermore, using Tarski-type theorems on extensions of invariant finitely additive measures, which are consequences of sandwich theorems, some applications are given, on constructing some invariant finitely additive vector lattice-valued probability measures defined on all subsets of suitable "product spaces" of the type $\Pi_{t \in \mathcal{T}} B_{t}$, where $\mathcal{T}$ can be viewed as the time space and the $B_{t}$ 's, $t \in \mathcal{T}$, can be interpreted as "events", e.g. as logical propositions about which one knows that at the instant $t$ (in the future or also in the past, for example in the case of lack of informations) they are true or false (see also [5]). The invariance allows to "replace" the study of the involved events related to some periods of time about which one has not enough knowledge with the corresponding events associated with other periods of time on which one has more informations.

\section{PRELIMINARIES}

We begin with giving the basic definitions and concepts, which will be useful for proving our results.

Let $X$ be a real vector space. An affine combination of elements $x_{1}, x_{2}, \ldots, x_{n}$ of $X$ is any linear combination of the form $\sum_{i=1}^{n} \lambda_{i} x_{i}$ with $\lambda_{1}, \lambda_{2}, \ldots, \lambda_{n} \in \mathbb{R}$ and $\sum_{i=1}^{n} \lambda_{i}=1$. An affine manifold of $X$ is a nonempty subset of $X$, closed under affine combinations.

If $\emptyset \neq Z \subset X$, then the affine hull of $Z$ is the smallest affine manifold of $X$ which contains $Z$, and we denote it by $\operatorname{span}_{\text {aff }}(Z)$ (see also [26]).

A point $x_{0} \in Z$ is an algebraic relative interior point of $Z$ iff for each $x \in \operatorname{span}_{\text {aff }}(Z)$ there is $\lambda_{0}>0$ with $(1-\lambda) x_{0}+\lambda x \in Z$ for each $\lambda \in]-\lambda_{0}, \lambda_{0}$ [. The set of all algebraic relative interior points of $Z$ is denoted by $\operatorname{int}(Z)$.

A nonempty subset $D$ of any real vector space $X$ is said to be convex iff $\lambda x_{1}+(1-\lambda) x_{2} \in D$ for every $x_{1}, x_{2} \in D$ and $\lambda \in[0,1]$.

Given any two real vector spaces $X, Y$ and a convex set $D \subset X$, we say that a function $U: X \rightarrow Y$ is convex on $D$ iff $U\left(\lambda x_{1}+\right.$ $\left.(1-\lambda) x_{2}\right) \leq \lambda U\left(x_{1}\right)+(1-\lambda) U\left(x_{2}\right)$ for every $x_{1}, x_{2} \in D$ and $\lambda \in[0,1]$. A function $U: D \rightarrow Y$ is said to be concave iff $-U$ is convex. We will set $D(U):=D$.

Let $G$ be a semigroup, and $\mathcal{P}(G)$ be the family of all subsets of $G$. We say that $G$ is left (resp. right) amenable) iff there is a finitely additive measure $\mu: \mathcal{P}(G) \rightarrow[0,1]$, with $\mu(G)=1$ and $\mu(\{h g: g \in E\})$ (resp. $\mu(\{g h: g \in E\})=\mu(E))$ for every $E \subset G$ and $g \in G$. We say that $G$ is amenable iff it is both left and right amenable. In general, left and right amenability are not equivalent, but, if $G$ a group, then they coincide (see also [18]).

Let $G \subset X^{X}$ be a right amenable semigroup of (linear) homomorphisms, with $(g h)(x)=g(h x)$ for any $g, h \in G$ and $x \in X, R$ be a Dedekind complete partially ordered vector space, $R^{+}:=\{y \in R: y \geq 0\}, l_{b}(G, R)$ be the space of all bounded $R$ valued functions defined on $G$. Given $f \in l_{b}(G, R)$ and $h \in G$, defined by ${ }_{h} f(g)=f(h g)$ (resp. $\left.f_{h}(g)=f(g h)\right), g \in G$. A left (resp. right)-G-invariant $R$-mean is a linear positive function $m: L^{\infty}(G) \rightarrow R$ such that $m\left({ }_{h} f\right)=m(f)\left(\operatorname{resp}\right.$. $m\left(f_{h}\right)=$ $m(f))$ for all $f \in l_{b}(G, R)$ and $h \in G$, and $m(\boldsymbol{y})=y$ for each $y \in R$, where $\boldsymbol{y}$ is the constant function which associates the value $y$ to every element $g \in G$.

A set $\emptyset \neq Z \subset X$ is $G$-invariant iff $g z \in Z$ whenever $z \in Z$. A set $\emptyset \neq A \subset X \times R$ is $G$-invariant iff $(g x, y) \in A$ whenever $g \in G$ and $(x, y) \in A$.

A function $L: X \rightarrow R$ is $G$-subinvariant (resp. G-superinvariant, $G$-invariant) iff $L(g x) \leq L(x)$ (resp. $L(g x) \geq L(x), L(g x)=$ $L(x))$ for every $g \in G$ and $x \in X$.

By $\mathcal{L}(X, R)$ and $\mathcal{L}(R, R)$ we denote the sets of all linear functions from $X$ to $R$ and from $R$ to $R$, respectively. We indicate with $\mathcal{L}_{\text {inv }}(X, R)$ the set of all linear $G$-invariant functions $L \in \mathcal{L}(X, R)$.

A nonempty set $A \subset X \times R$ is called a cone with vertex $x_{0} \in X \times R$ iff $\lambda\left(A-x_{0}\right) \subset A-x_{0}$ for every positive real number $\lambda$. Sometimes we associate with $X$ a $G$-invariant cone $X^{+} \subset X$ with vertex 0 , and the corresponding order on $X$ defined by $x_{1} \geq x_{2}$ if and only if $x_{1}-x_{2} \in X^{+}$. In this context we will always require that $g x_{1} \geq g x_{2}$ whenever $g \in G$ and $x_{1} \geq x_{2}$, without saying it explicitly. If $X$ has such a cone $X^{+}$, then we say that $X^{+}$has property $\left.\mathcal{K}\right)$, and denote by $\mathcal{L}_{+, \text {inv }}(X, R)$ the set of all positive functions $L \in \mathcal{L}_{\text {inv }}(X, R)$.

Given $0 \not \equiv L \in \mathcal{L}(X, R), 0 \not L^{\prime} \in \mathcal{L}(X, R)$ and $u_{0}$ in $R$, set

$$
H:=\left\{(x, y) \in X \times R: L(x)+L^{\prime}(y)=u_{0}\right\} .
$$

It is not difficult to check that the set $H$ defined in (1) is empty or an affine manifold of $X \times R$ (see also [34]). 
If $A, B$ are two nonempty subsets of $X \times R$ and $H \neq \emptyset$ is as in (1), then we say that $H$ separates $A$ and $B$ iff $A \subset H^{-}$and $B \subset H^{+}$, where

$$
H^{-}:=\left\{(x, y) \in X \times R: L(x)+L^{\prime}(y) \leq u_{0}\right\}, H^{-}:=\left\{(x, y) \in X \times R: L(x)+L^{\prime}(y) \geq u_{0}\right\} .
$$

The projection of $X \times R$ onto $X$ is the function $P_{X}: X \times R \rightarrow X$ defined by $P_{X}(x, y)=x$ for every $(x, y) \in X \times R \rightarrow X$. Moreover, for any nonempty set $A \subset X \times R$, put

$$
P_{X}(A):=\{x \in X: \text { there exists } y \in R \text { with }(x, y) \in A\} .
$$

It is not difficult to see that $P_{X}(A+B)=P_{X}(A)+P_{X}(B)$ for any two nonempty subsets $A, B \subset X \times R$.

Given a set $\emptyset \neq A \subset X \times R$, we call cone generated by $A$ the set $C(A):=\left\{a \in X \times R\right.$ : there exist $\lambda \in \mathbb{R}_{0}^{+}$and $a^{\prime} \in A$ with $\left.a=\lambda a^{\prime}\right\}$. It is not difficult to check that, if $A$ is convex, then $C(A)$ is too, and that $A-B$ and $C(A-B)$ are $G$-invariant whenever $A$ and $B$ are $G$-invariant.

In proving our main results, we often will do the following assumption:

$\mathcal{H}$ ) let $U: D(U) \rightarrow R, V: D(V) \rightarrow R$ be two convex and $G$-subinvariant functions, where $D(U)$ and $D(V)$ are nonempty convex and $G$-invariant subsets of $X$ with $\operatorname{int}(D(U)) \cap \operatorname{int}(D(V)) \neq \emptyset$,

and we will put $P_{U, V}:=D(U) \cap D(V)$.

The $G$-invariant conjugate (shortly, conjugate) of $U$ is the $R$-valued function $U^{c}$ defined as

$$
U^{c}(L):=\bigvee\{L(x)-U(x): x \in D(U)\}, \quad L \in D\left(U^{c}\right)
$$

where

$$
D\left(U^{c}\right):=\left\{L \in \mathcal{L}_{\mathrm{inv}}(X, R): \bigvee\{L(x)-U(x): x \in D(U)\}\right.
$$

exists in $R\}$,

provided that $D\left(U^{c}\right) \neq \emptyset$. If $x_{0} \in D(U)$, then we call $G$-invariant subdifferential (shortly, subdifferential) at $x_{0}$ the set $\partial_{\text {inv }} U\left(x_{0}\right)$ defined as

$$
\partial_{\mathrm{inv}} U\left(x_{0}\right):=\left\{L \in \mathcal{L}_{\mathrm{inv}}(X, R): L(x)-L\left(x_{0}\right) \leq U(x)-U\left(x_{0}\right)\right\} .
$$

Any element $L \in \partial_{\text {inv }} U\left(x_{0}\right)$ will be called ( $G$-invariant) subgradient of $U$ at $x_{0}$

Given a nonempty set $A \subset X$ and $x_{0} \in X$, we call $G$-invariant polar (in brief, polar) of $A$ at $x_{0}$ the set $A_{\mathrm{inv}}^{*}\left(x_{0}\right):=\left\{L \in \mathcal{L}_{\mathrm{inv}}(X, R): L(x)-L\left(x_{0}\right) \leq 0\right.$ for every $\left.x \in A\right\}$.

In the study of Fenchel-type duality theorem, we will consider the following programs (see also [45]).

Program I. Find $r:=\Lambda\left\{U(x)+V(x): x \in P_{U, V}\right\}$ in $R$.

Program II. Find $s:=\mathrm{V}\left\{-U^{c}(L)-V^{c}(-L): L \in D\left(U^{c}\right) \cap D\left(V^{c}\right)\right\}$ in $R$, provided that $D\left(U^{c}\right) \cap D\left(V^{c}\right) \neq \emptyset$.

\section{THE MAIN RESULTS}

We begin with the following sandwich theorem in the setting of invariance with respect to amenable semigroups of transformations and partially ordered vector spaces, extending [47, Sandwich Theorem 3.1]. Our technique is based on the existence of linear operators, not necessarily invariant, due to the corresponding classical results, and of suitable invariant partially ordered vector space-valued means (see also [15, Théorème 2]), from which it is possible to construct invariant linear operators.

Theorem 3.1. (Sandwich theorem) Let $U: D(U) \rightarrow R$ and $V: D(V) \rightarrow R$ satisfy assumption $\mathcal{H})$. Assume that $U(x)+V(x) \geq$ 0 for every $x \in P_{U, V}$. Then there exist $L \in \mathcal{L}_{\text {inv }}(X, R)$ and $u_{0} \in R$ with $L(x)-u_{0} \leq U(x)$ for every $x \in D(U)$ and $L(x)-u_{0} \geq$ $-V(x)$ for each $x \in D(V)$.

Proof. By [47, Proposition 3.9 (b)], we get $0 \in \operatorname{int}(D(U)-D(V))$. By [47, Sandwich Theorem 3.1], there are an element $u_{0} \in R$ and a function $L^{*} \in \mathcal{L}(X, R)$ (not necessarily $G$-invariant) with $L^{*}(x)-u_{0} \leq U(x)$ for every $x \in D(U)$ and $L^{*}(x)-u_{0} \geq$ $-V(x)$ for each $x \in D(V)$. Pick arbitrarily $x \in X$, and define $f_{x} \in l_{b}(G, R)$ by $f_{x}(g)=L^{*}(g x), g \in G$. As $R$ is Dedekind complete and $G$ is right amenable, by [15, Théorème 2] there is a right $G$-invariant $R$-mean $m: l_{b}(G, R) \rightarrow R$. Set $L(x):=$ $m\left(f_{x}\right), x \in X$. Since $f_{h x}(g)=L^{*}(g h x)=f_{x}(g h)=\left(f_{x}\right)_{h}(g)$ for any $g \in G$, then $L(h x)=m\left(f_{h x}\right)=m\left(\left(f_{x}\right)_{h}\right)=m\left(f_{x}\right)=L(x)$ for every $h \in G$, and hence $L$ is $G$-invariant. As $D(U)$ and $D(V)$ are $G$-invariant, $U$ and $V$ are $G$-subinvariant, $L^{*}(x) \leq u_{0}+$ $U(x)$ for every $x \in D(U)$ and $L^{*}(x) \geq u_{0}-V(x)$ for each $x \in D(V)$, then $f_{x}(g)=L^{*}(g x) \leq u_{0}+U(g x) \leq u_{0}+U(x)$ for every $x \in D(U)$ and $g \in G$, and hence

$$
L(x)=m\left(f_{x}\right) \leq m\left(u_{\mathbf{0}}+\boldsymbol{U}(\boldsymbol{x})\right)=m\left(u_{\mathbf{0}}\right)+m(\boldsymbol{U}(\boldsymbol{x}))=u_{0}+U(x)
$$

for any $x \in D(U)$. Analogously it is possible to prove that $L(x) \geq u_{0}-V(x)$ for every $x \in D(V)$. Moreover, if $\lambda_{1}, \lambda_{2} \in \mathbb{R}, x_{1}$, $x_{2} \in X$ and $g \in G$, then

and therefore

$$
\begin{aligned}
& f_{\lambda_{1} x_{1}+\lambda_{2} x_{2}}(g)=L^{*}\left(g\left(\lambda_{1} x_{1}+\lambda_{2} x_{2}\right)\right)=L^{*}\left(\lambda_{1} g x_{1}+\lambda_{2} g x_{2}\right)= \\
& =\lambda_{1} L^{*}\left(g x_{1}\right)+\lambda_{2} L^{*}\left(g x_{2}\right)=\lambda_{1} f_{x_{1}}(g)+\lambda_{2} f_{x_{2}}(g),
\end{aligned}
$$

$$
L\left(\lambda_{1} x_{1}+\lambda_{2} x_{2}\right)=m\left(f_{\lambda_{1} x_{1}+\lambda_{2} x_{2}}\right)=
$$


Thus, $L \in \mathcal{L}_{\text {inv }}(X, R)$. This completes the proof

$$
=\lambda_{1} m\left(f_{x_{1}}\right)+\lambda_{2} m\left(f_{x_{2}}\right)=\lambda_{1} L\left(x_{1}\right)+\lambda_{2} L\left(x_{2}\right) .
$$

The next step is to prove the following theorems and their equivalence with Theorem 3.1, extending to invariance [22, Theorems 1 and 2].

Theorem 3.2. (Fenchel duality theorem) Under the assumption $\mathcal{H})$, suppose that $r:=\Lambda\left\{U(x)+V(x): x \in P_{U, V}\right\}$ exists in $R$. Then Program II. has a solution $L_{0}$, and $-U^{c}\left(L_{0}\right)-V^{c}\left(-L_{0}\right)=r$.

Theorem 3.3. Under the assumption $\mathcal{H}$ ), let $x_{0} \in P_{U, V}$ be a solution of Program I. Then

$$
\partial_{\text {inv }} U\left(x_{0}\right) \cap\left(-\partial_{\text {inv }} V\left(x_{0}\right)\right) \neq \emptyset .
$$

Theorem 3.4. Under the assumption $\mathcal{H})$, let $x_{0} \in P_{U, V}, \partial_{\text {inv }} U\left(x_{0}\right) \neq \varnothing$ and $\partial_{\text {inv }} V\left(x_{0}\right) \neq \emptyset$. Then

$$
\partial_{\mathrm{inv}}(U+V)\left(x_{0}\right)=\partial_{\mathrm{inv}}(U)\left(x_{0}\right)+\partial_{\mathrm{inv}}(V)\left(x_{0}\right) .
$$

Theorem 3.5. Let $A_{1}, A_{2} \subset X$ be convex $G$-invariant sets with $\left(\operatorname{int}\left(A_{1}\right)\right) \cap\left(\operatorname{int}\left(A_{2}\right)\right) \neq \emptyset$. Then

for every $x_{0} \in A_{1} \cap A_{2}$.

$$
\left(A_{1}\right)_{\mathrm{inv}}^{*}\left(x_{0}\right)+\left(A_{2}\right)_{\mathrm{inv}}^{*}\left(x_{0}\right)=\left(A_{1} \cap A_{2}\right)_{\mathrm{inv}}^{*}\left(x_{0}\right)
$$

Theorem 3.6. (Hahn-Banach) Let $U: D(U) \rightarrow R$ be convex and $G$-subinvariant, let $D(U)$ be $G$-invariant, $U(0)=0$, and $Z \subset X$ be a $G$-invariant subspace. Assume that $\operatorname{int}(D(U)) \cap Z \neq \emptyset$. Let $L_{0} \in \mathcal{L}_{\text {inv }}(Z, R)$ be such that $L_{0}(z) \leq U(z)$ for every $z \in D(U) \cap Z$.

Then $L_{0}$ has an extension $L \in \mathcal{L}_{\text {inv }}(X, R)$, such that $L(x) \leq U(x)$ for each $x \in D(U)$.

Theorem 3.7. Let $U$ and $D(U)$ be as in Theorem 3.6. If $0 \in \operatorname{int}(D(U))$ and $U(0)=0$, then there exists $L \in \mathcal{L}_{\mathrm{inv}}(X, R)$, with $L(x) \leq U(x)$ for each $x \in D(U)$.

Theorem 3.8. Let $U$ and $D(U)$ be as in Theorem 3.6. If $0 \in \operatorname{int}(D(U))$ and $U(0) \geq 0$, then there is $L \in \mathcal{L}_{\text {inv }}(X, R)$, such that $L(x) \leq U(x)$ for each $x \in D(U)$.

Theorem 3.9. (Krein) Let $X^{+} \subset X$ be a $G$-invariant cone satisfying property $\mathcal{K}$ ), $Z \subset X$ be a $G$-invariant subspace with the order generated by $Z \cap X^{+}$, such that $\operatorname{int}\left(X^{+}\right) \cap Z \neq \emptyset$, and $L_{0} \in \mathcal{L}_{+, \text {inv }}(Z, R)$. Then there is $L \in \mathcal{L}_{+, \text {inv }}(Z, R)$, with $L(z)=$ $L_{0}(z)$ for every $x \in Z$.

Theorem 3.10. (Separation theorem) Let $A, B$ be two $G$-invariant subsets of $X \times R$ such that $C(A-B)$ is convex,

$$
\operatorname{int}\left(P_{X}(A)\right) \cap \operatorname{int}\left(P_{X}(B)\right) \neq \emptyset
$$

and

$$
y_{1} \geq y_{2} \text { whenever }\left(x, y_{1}\right) \in A \text { and }\left(x, y_{2}\right) \in B \text {. }
$$

Then there exist $L \in \mathcal{L}_{\text {inv }}(X, R)$ and $u_{0} \in R$ such that the affine manifold

$$
H:=\left\{(x, y) \in R \times X: L(x)-y=u_{0}\right\}
$$

separates $A$ and $B$.

Theorem 3.11. (Farkas) Let $U: D(U) \rightarrow X$ be convex and $G$-equivariant (that is, $U(g x)=g(U(x)$ ) for every $g \in G$ and $x \in D(U)$, see also [13]) $V: D(V) \rightarrow R$ be convex and $G$-invariant, $D(U)$ and $D(V)$ be $G$-invariant, assume that $X_{0}:=D(U) \cap$ $D(V) \neq \emptyset, 0 \in \operatorname{int}\left(U\left(X_{0}\right)+X^{+}\right)$and

$$
V(x) \geq 0 \text { whenever } x \in X_{0} \text { and } U(x) \leq 0 \text {. }
$$

Then there is $L \in \mathcal{L}_{+, \mathrm{inv}}(X, R)$ with

$$
V(x)+L(U(x)) \geq 0 \text { whenever } x \in X_{0} .
$$

Theorem 3.12. (Kuhn-Tucker) Under the same hypotheses and notations as in Theorem 3.11, let $x_{0}$ be a solution of the problem

P1) find $x_{0} \in Z_{0}:=\left\{x \in X_{0}: U(x) \leq 0\right\}$ such that $V\left(x_{0}\right) \leq V(x)$ for every $x \in Z_{0}$.

Then there exists $L_{0} \in \mathcal{L}_{+, i n v}(X, R)$ such that $\left(x_{0}, L_{0}\right)$ is a solution of the problem

P2) find $x_{0} \in X_{0}$ and $L_{0} \in \mathcal{L}_{+, i n v}(X, R)$ such that

$$
L\left(U\left(x_{0}\right)\right)+V\left(x_{0}\right) \leq L_{0}\left(U\left(x_{0}\right)\right)+V\left(x_{0}\right) \leq L_{0}(U(x))+V(x),
$$

for every $x \in X_{0}$ and $L \in \mathcal{L}_{+, i n v}(X, R)$.

Theorem 3.13. Let $X^{+} \subset X$ be a $G$-invariant cone having property $\left.\mathcal{K}\right), Z \subset X$ be a $G$-invariant subspace, $L_{1} \in \mathcal{L} \quad(Z, X), L_{1}$ be $G$-equivariant, and $L_{2} \in \mathcal{L}_{\text {inv }}(Z, R)$. Suppose that $L_{1}(Z) \cap \operatorname{int}\left(-X^{+}\right) \neq \emptyset$, and

$$
L_{2}(x) \geq 0 \text { whenever } x \in Z \text { and } L_{1}(x) \leq 0 .
$$


Then there is $L_{0} \in \mathcal{L}_{+, \text {inv }}(X, R)$ such that

$$
L_{0}\left(L_{1}(x)\right)+L_{2}(x)=0 \text { whenever } x \in Z \text {. }
$$

First of all, we prove the implications (3.1) $\Rightarrow$ (3.2) $\Rightarrow$ (3.3) $\Rightarrow(3.6) \Rightarrow$ (3.7) $\Rightarrow$ (3.8) $\Rightarrow$ (3.1).

(3.1) $\Rightarrow$ (3.2) We extend to invariance [45, Theorem 2]. Let $\widetilde{U}(x)=U(x)-r$, where $r:=\Lambda\left\{U(x)+V(x): x \in P_{U, V}\right\}$. By hypothesis, the element $r$ exists in $R$. It is not difficult to check that $\widetilde{U}$ is convex, since $U$ is. Moreover, $P_{\widetilde{U}, V}:=D(\widetilde{U}) \cap$ $D(V)=D(U) \cap D(V)=P_{U, V}$. For each $x \in P_{U, V}$ it is $r \leq U(x)-V(x)$, and hence $\widetilde{U}(x)+V(x) \geq 0$. Thus, $\widetilde{U}$ and $V$ satisfy the hypotheses of Theorem 3.1. Therefore, there exist $L_{0} \in \mathcal{L}_{\text {inv }}(X, R)$ and $u_{0} \in R$ with $L_{0}(x)-u_{0} \leq \widetilde{U}(x)=U(x)-r$ for every $x \in D(U)$ and $L_{0}\left(x^{\prime}\right)-u_{0} \geq-V\left(x^{\prime}\right)$ for each $x^{\prime} \in D(V)$. From this we get

$$
L_{0}\left(x-x^{\prime}\right)=L_{0}(x)-L_{0}\left(x^{\prime}\right) \leq u_{0}+U(x)-r-u_{0}+V\left(x^{\prime}\right)
$$

and hence

$$
r+L_{0}(x)-U(x) \leq L_{0}\left(x^{\prime}\right)+V\left(x^{\prime}\right)
$$

for each $x \in D(U)$ and $x^{\prime} \in D(V)$. Thus,

$$
\begin{aligned}
r & +\bigvee\left\{L_{0}(x)-U(x): x \in D(U)\right\} \leq \bigwedge\left\{L_{0}(x)+V(x): x \in D(V)\right\} \\
& =-\bigvee\left\{-L_{0}(x)-V(x): x \in D(V)\right\},
\end{aligned}
$$

and hence $L_{0} \in D\left(U^{c}\right) \cap D\left(V^{c}\right)$ and $r \leq-U^{c}\left(L_{0}\right)-V^{c}\left(-L_{0}\right)$. Furthermore, observe that

$$
-U^{c}(L)-V^{c}(-L) \leq-L(x)+U(x)+L(x)+V(x)=U(x)+V(x)
$$

for every $x \in P_{U, V}$ and $L \in D\left(U^{c}\right) \cap D\left(V^{c}\right)$. Taking the infimum as $x \in P_{U, V}$ and the supremum as $L \in D\left(U^{c}\right) \cap D\left(V^{c}\right)$, we obtain

$$
r \leq-U^{c}\left(L_{0}\right)-V^{c}\left(-L_{0}\right) \leq s:=\bigvee\left\{-U^{c}(L)-V^{c}(-L): L \in D\left(U^{c}\right) \cap D\left(V^{c}\right)\right\} \leq r .
$$

Thus, $r=-U^{c}\left(L_{0}\right)-V^{c}\left(-L_{0}\right)=s$, and the supremum in Program II. is a maximum, attained by $L_{0}$. So, the assertion follows.

Remark 3.14. Observe that, in general, the converse of Theorem 3.2 does not hold (see also [45]).

(3.2) $\Rightarrow$ (3.3) We characterize the solutions of Program I. in terms of $G$-invariant subgradients, extending to invariance [45, Theorem 3]. Let $x_{0}$ be a solution of Program I. Then, by Theorem 2, Program II. has a solution $L_{0}$, that is an element $L_{0} \in \mathcal{L}_{\text {inv }}(X, R)$ with

$$
U\left(x_{0}\right)+V\left(x_{0}\right)=-U^{c}\left(L_{0}\right)-V^{c}\left(-L_{0}\right) .
$$

By definition of the conjugate function, from (12) we get

$$
U\left(x_{0}\right)+V\left(x_{0}\right) \leq U(x)+V\left(x^{\prime}\right)-L_{0}(x)+L_{0}\left(x^{\prime}\right)
$$

for every $x \in D(U)$ and $x^{\prime} \in D(V)$. From (13) used with $x=x_{0}$ and $x^{\prime}=x_{0}$ we obtain $-L_{0} \in \partial_{\text {inv }} V\left(x_{0}\right)$ and $L_{0} \in \partial_{\text {inv }} U\left(x_{0}\right)$, respectively.

Conversely, let $L \in \partial_{\text {inv }} U\left(x_{0}\right) \cap\left(-\partial_{\text {inv }} V\left(x_{0}\right)\right)$. Then for every $x \in P_{U, V}$ it is

$$
U(x)-U\left(x_{0}\right) \geq L(x)-L\left(x_{0}\right) \geq V\left(x_{0}\right)-V(x)
$$

and hence $U(x)+V(x) \geq U\left(x_{0}\right)+V\left(x_{0}\right)$. Thus, $x_{0}$ is a solution of Program I.

(3.3) $\Rightarrow$ (3.6) We extend to invariance [47, Theorem 1]. Let $Z, L_{0}, U$ be as in the hypotheses of Theorem 3.6. Set $V:=$ $-L_{0}$. Then $D(V)=D\left(L_{0}\right)=Z$. Since $L_{0}(z) \leq U(z)$ for each $z \in D(U) \cap Z$ and $L_{0}(0)=U(0)=0$, then we get

$$
0=U(0)+V(0)=\min \{U(x)+V(x): x \in D(U) \cap Z\} .
$$

By Theorem 3.3 there is $L \in \partial_{\text {inv }} U\left(x_{0}\right) \cap\left(-\partial_{\text {inv }} V\left(x_{0}\right)\right)$. We have

$$
L(x)=L(x)-L(0) \leq U(x)-U(0)=U(x)
$$

for each $x \in D(U)$ and

$$
-L(z)=-L(z)+L(0) \leq-L_{0}(z)+L_{0}(0)=-L_{0}(z),
$$

that is

$$
L(z) \geq L_{0}(z)
$$

for each $z \in Z$. Taking $-z$ in (14) (note that $-z \in Z$ ), we obtain 


$$
L(z)=-L(-z) \leq-L_{0}(-z)=L_{0}(z)
$$

for every $z \in Z$. From (15) and (16) it follows that $L(z)=L_{0}(z)$ for all $z \in Z$. Thus, the assertion follows.

(3.6) $\Rightarrow$ (3.7) It is enough to take $Z:=\{0\}$ in Theorem 3.6.

$(3.7) \Rightarrow$ (3.8) We extend to invariance [47, Corollary 2.6]. Set $\widetilde{U}(x):=U(x)-U(0)$. Since $\widetilde{U}(0)=0, \widetilde{U}$ satisfies the hypotheses of Theorem 3.7. Therefore, there exists $L \in \mathcal{L}_{\text {inv }}(X, R)$ with $L(x) \leq \widetilde{U}(x) \leq U(x)$ for every $x \in D(U)$, since $U(0) \geq 0$. This ends the proof.

(3.8) $\Rightarrow$ (3.1) Proceeding similarly as in [47, Sandwich Theorem 3.1], let $\mathcal{D}:=D(U)-D(V), \mathcal{E}:=\left\{\left(x_{1}-x_{2}, U\left(x_{1}\right)+V\left(x_{2}\right)+\right.\right.$ $\left.y) \in X \times R: x_{1} \in D(U), x_{2} \in D(V), y \geq 0\right\}$, and $\varepsilon_{x}:=\{y \in R:(x, y) \in \mathcal{E}\}$. Since $U$ and $V$ are convex, then $\mathcal{E}$ is convex. Moreover $\mathcal{E}$ is $G$-invariant, because $D(U)$ and $D(V)$ are $G$-invariant. Furthermore, note that $\varepsilon_{0} \subset R^{+}:$indeed, if $y \in \mathcal{E}_{0}$, then $x_{1}=x_{2}$, and hence $U\left(x_{1}\right)+V\left(x_{2}\right)+y \geq 0$.

Now define $p: \mathcal{E} \rightarrow R$ by $p(x):=\wedge \varepsilon_{x}, x \in \mathcal{D}$. We show that $p$ is well-defined. Fix $x \in \mathcal{E}$. Since $0 \in \operatorname{int}(\mathcal{E})$ (see also [47]), there is $\lambda \in] 0,1]$ small enough with $-\lambda x \in \mathcal{E}$. Pick $y^{\prime} \in \mathcal{E}_{-\lambda x}$. Since $\mathcal{E}$ is convex, then for every $y \in \mathcal{E}_{x}$ it is

$$
\left(0, \frac{1}{1+\lambda} y^{\prime}+\frac{\lambda}{1+\lambda} y\right)=\frac{1}{1+\lambda}\left(-\lambda x, y^{\prime}\right)+\frac{\lambda}{1+\lambda}(x, y) \in \mathcal{E},
$$

that is $\frac{1}{1+\lambda} y^{\prime}+\frac{\lambda}{1+\lambda} y \in \varepsilon_{0}$, and hence $\frac{1}{1+\lambda} y^{\prime}+\frac{\lambda}{1+\lambda} y \geq 0$, since $\varepsilon_{0} \subset R^{+}$. Thus, $\wedge \varepsilon_{x}$ exists in $R$, and hence $p$ is well-defined. Now we claim that $p$ is convex. If $y_{1} \in \mathcal{E}_{x_{1}}, y_{2} \in \mathcal{E}_{x_{2}}$ and $\lambda \in[0,1]$, then $\left(x_{1}, y_{1}\right) \in \mathcal{E},\left(x_{2}, y_{2}\right) \in \mathcal{E}$, and by convexity of $\mathcal{E}$ we get $\left(\lambda x_{1}+(1-\lambda) x_{2}, \lambda y_{1}+(1-\lambda) y_{2}\right) \in \mathcal{E}$. Thus,

$$
\lambda y_{1}+(1-\lambda) y_{2} \in \mathcal{E}_{\lambda x_{1}+(1-\lambda) x_{2}} .
$$

From (17) and arbitrariness of $y_{1}$ and $y_{2}$ we obtain

$$
\bigwedge \varepsilon_{\lambda x_{1}+(1-\lambda) x_{2}} \leq \lambda \bigwedge \varepsilon_{x_{1}}+(1-\lambda) \bigwedge \varepsilon_{x_{2}}
$$

that is convexity of $p$.

Furthermore, note that $p(0) \geq 0$ because $\varepsilon_{0} \subset R^{+}$, and, since $D(U)$ and $D(V)$ are $G$-invariant, we get $(g x, y) \in \mathcal{E}$ whenever $(x, y) \in \mathcal{E}$ and $g \in G$, namely $y \in \mathcal{E}_{g x}$ whenever $(x, y) \in \mathcal{E}$ and $g \in G$. Hence, $\wedge \varepsilon_{g x} \leq \wedge \varepsilon_{x}$ for each $x \in \mathcal{D}$ and $g \in G$. Thus, $p$ is $G$-subinvariant.

By Theorem 3.8 there exists $L \in \mathcal{L}_{\text {inv }}(X, R)$ such that

and hence

$$
L\left(x-x^{\prime}\right) \leq p\left(x-x^{\prime}\right)=\bigwedge \varepsilon_{x-x^{\prime}} \leq U(x)+V\left(x^{\prime}\right),
$$

$$
L(x)-U(x) \leq L\left(x^{\prime}\right)+V\left(x^{\prime}\right)
$$

for any $x \in D(U)$ and $x^{\prime} \in D(V)$. Set

$$
u_{0}:=\bigwedge\left\{L\left(x^{\prime}\right)+V\left(x^{\prime}\right): x^{\prime} \in D(V)\right\} .
$$

Note that $u_{0} \in R$, since $R$ is Dedekind complete. From (18) we get $L(x)-u_{0} \leq U(x)$ for each $x \in D(U)$, and from (19) we obtain $L\left(x^{\prime}\right)-u_{0} \geq-V\left(x^{\prime}\right)$ for every $x^{\prime} \in D(V)$. Thus, the assertion follows.

Now we prove the implications (3.2) $\Rightarrow$ (3.4) $\Rightarrow$ (3.5) $\Rightarrow$ (3.9)

$(3.2) \Rightarrow$ (3.4) We extend [45, Theorem 4] to $G$-invariant subdifferentials. Choose arbitrarily $L^{\prime} \in \partial_{\text {inv }}(U+V)\left(x_{0}\right)$, and set $V^{\prime}(x)=V(x)-L^{\prime}(x), x \in D(V)$. It is not difficult to check that $V^{\prime}$ is convex. Moreover, by definition of subdifferential, it is

$$
L^{\prime}(x)-L^{\prime}\left(x_{0}\right) \leq(U+V)(x)-(U+V)\left(x_{0}\right),
$$

and hence

$$
U\left(x_{0}\right)+V\left(x_{0}\right)-L^{\prime}\left(x_{0}\right) \leq U(x)+V(x)-L^{\prime}(x)=U(x)+V^{\prime}(x)
$$

for every $x \in D(U) \cap D(V)$.

Furthermore, it is

$$
\left(V^{\prime}\right)^{c}(-L)=\bigvee\left\{L^{\prime}(x)-L(x)-V(x): x \in D(V)\right\}=V^{c}\left(L^{\prime}-L\right)
$$

for every $L \in D\left(\left(V^{\prime}\right)^{c}\right)=D\left(V^{c}\right)-L^{\prime}$.

By (20) and Theorem 3.2, there exists $L_{0} \in \mathcal{L}_{\text {inv }}(X, R)$, with

$$
U\left(x_{0}\right)+V\left(x_{0}\right)-L^{\prime}\left(x_{0}\right)=-U^{c}\left(L_{0}\right)-\left(V^{\prime}\right)^{c}\left(-L_{0}\right)=-U^{c}\left(L_{0}\right)-V^{c}\left(L^{\prime}-L_{0}\right),
$$

that is 


$$
U^{c}\left(L_{0}\right)+V^{c}\left(L^{\prime}-L_{0}\right)=L_{0}\left(x_{0}\right)-U\left(x_{0}\right)+\left(L^{\prime}-L_{0}\right)\left(x_{0}\right)-V\left(x_{0}\right) .
$$

By definition of conjugate function, it is

$$
\begin{aligned}
& U^{c}\left(L_{0}\right) \geq L_{0}\left(x_{0}\right)-U\left(x_{0}\right), \\
& V^{c}\left(L^{\prime}-L_{0}\right) \geq L^{\prime}\left(x_{0}\right)-L_{0}\left(x_{0}\right)-V\left(x_{0}\right) .
\end{aligned}
$$

From (21) and (22) it follows that

and hence

$$
U^{c}\left(L_{0}\right)=L_{0}\left(x_{0}\right)-U\left(x_{0}\right), V^{c}\left(L^{\prime}-L_{0}\right)=L^{\prime}\left(x_{0}\right)-L_{0}\left(x_{0}\right)-V\left(x_{0}\right),
$$

$$
L_{0}(x)-U(x) \leq L_{0}\left(x_{0}\right)-U\left(x_{0}\right) \text { for every } x \in D(U)
$$

$$
L^{\prime}(x)-L_{0}(x)-V(x) \leq L^{\prime}\left(x_{0}\right)-L_{0}\left(x_{0}\right)-V\left(x_{0}\right) \text { for every } x \in D(V) .
$$

Thus, $L_{0} \in \partial_{\mathrm{inv}}(U)\left(x_{0}\right), L^{\prime}-L_{0} \in \partial_{\mathrm{inv}}(V)\left(x_{0}\right)$, and hence $L^{\prime} \in \partial_{\mathrm{inv}}(U)\left(x_{0}\right)+\partial_{\mathrm{inv}}(V)\left(x_{0}\right)$. By arbitrariness of $L^{\prime}$, it follows that

$$
\partial_{\mathrm{inv}}(U+V)\left(x_{0}\right) \subset \partial_{\mathrm{inv}}(U)\left(x_{0}\right)+\partial_{\mathrm{inv}}(V)\left(x_{0}\right) .
$$

The proof of the converse inclusion is straightforward.

(3.4) $\Rightarrow$ (3.5) Let $A_{1}, A_{2} \subset X$ be convex $G$-invariant sets with $\left(\operatorname{int}\left(A_{1}\right)\right) \cap\left(\operatorname{int}\left(A_{2}\right)\right) \neq \emptyset$, let $A_{0}:=A_{1} \cap A_{2}$, and set $\mathcal{N}_{A_{j}}(x):=$ 0 for each $x \in A_{j}, j=0,1,2$. It is not difficult to see that

$$
\partial_{\mathrm{inv}} \mathcal{N}_{A_{j}}(x)=\left(A_{j}\right)_{\mathrm{inv}}^{*}(x) \text { for each } x \in A_{j}, j=0,1,2 \text {, }
$$

and

$$
\mathcal{N}_{A_{0}}(x)=\mathcal{N}_{A_{1}}(x)+\mathcal{N}_{A_{2}}(x) \text { for each } x \in A_{0} .
$$

Let now $x_{0} \in A_{0}$ be fixed. From (4), (23) and (24) we get

So, the assertion follows.

$$
\begin{aligned}
& \left(A_{1}\right)_{\text {inv }}^{*}\left(x_{0}\right)+\left(A_{2}\right)_{\text {inv }}^{*}\left(x_{0}\right)=\partial_{\text {inv }} \mathcal{N}_{A_{1}}\left(x_{0}\right)+\partial_{\text {inv }} \mathcal{N}_{A_{2}}\left(x_{0}\right)= \\
& =\partial_{\text {inv }}\left(\mathcal{N}_{A_{1}}+\mathcal{N}_{A_{2}}\right)\left(x_{0}\right)=\partial_{\text {inv }} \mathcal{N}_{A_{0}}\left(x_{0}\right)=\left(A_{0}\right)_{\text {inv }}^{*}\left(x_{0}\right) .
\end{aligned}
$$

(3.5) $\Rightarrow$ (3.9) We extend to invariance [47, Corollary 3.11]. Let $X^{+}, Z$ and $L_{0}$ be as in the hypotheses of Theorem 3.9. Let $Z^{\prime}$ be an algebraic complement of $Z$, namely a subspace of $X$ with the property that every $x \in X$ can be uniquely represented as $x=z+z^{\prime}$, where $z \in Z, z^{\prime} \in Z^{\prime}$. Such a subspace does exist, thanks to [29, Theorem 7.3.3]. Let us define $L_{1}: X \rightarrow R$ by $L_{1}(x)=-L_{0}(z), x \in X$. Since $L_{0} \in \mathcal{L}_{+, \text {inv }}(Z, R)$, then $L_{1} \in\left(X^{+} \cap Z\right)^{*}(0)$. By applying Theorem 3.5 with $x_{0}=0$, we find two functions $L, L^{\prime} \in \mathcal{L}_{\text {inv }}(X, R)$ with $L_{1}(x)=L(x)+L^{\prime}(x)$ for every $x \in X, L^{\prime}(x) \leq 0$ for each $x \in X^{+}$and $L(z) \leq 0$ for any $z \in Z$. Since $-z \in Z$ whenever $z \in Z$, it follows that $-L(z)=L(-z) \leq 0$, and hence $L(z)=0$ for all $z \in Z$. If $L^{*}:=$ $-L^{\prime}$, then $L^{*} \in \mathcal{L}_{+, \text {inv }}(X, R)$ and $L^{*}(z)=L_{0}(z)$ for every $z \in Z$

The proof of $(3.8) \Rightarrow(3.10)$ is given in [6, Theorem 3.4] (see also [47, Proposition 3.9]).

We now prove (3.10) $\Rightarrow(3.6)$. Let $Z$ be any $G$-invariant subspace of $X, L_{0} \in \mathcal{L}_{\text {inv }}(Z, R), U: D(U) \rightarrow R$ be a convex and $G$ subinvariant function, with $U(0)=0$ and $L_{0}(z) \leq U(z)$ for every $z \in Z$. Let

$$
\begin{gathered}
A:=\text { epi } U:=\{(x, y) \in X \times R, y \geq U(x)\}, \\
B:=\text { hypo } L_{0}:=\left\{(x, y) \in Z \times R, y \leq L_{0}(x)\right\} .
\end{gathered}
$$

It is not difficult to see that $A$ and $B$ are nonempty convex $G$-invariant subsets of $X \times R$, because $U$ is convex and $G$ subinvariant, and $L_{0}$ is linear and $G$-invariant. The sets $A$ and $B$ are called the epigraph of $U$ and the hypograph of $L_{0}$, respectively. Moreover, we get $P_{X}(A)=X, P_{X}(B)=X$ and

$$
\operatorname{int}\left(P_{X}(A)\right) \cap \operatorname{int}\left(P_{X}(B)\right)=\operatorname{int}(X) \cap \operatorname{int}(X)=X
$$

(see also [21, Satz 1 (6)]). Furthermore, if $\left(x, y_{1}\right) \in A,\left(x, y_{2}\right) \in B$, then $x \in Z$, and $y_{1} \geq U(x) \geq L_{0}(x) \geq y_{2}$.

By virtue of Theorem 3.10, there exist a function $L \in \mathcal{L}_{\text {inv }}(X, R)$ and an element $u_{0} \in R$ with $L\left(x_{1}\right)-y_{1} \leq u_{0} \leq L\left(x_{2}\right)-y_{2}$ for each $\left(x_{1}, y_{1}\right) \in A$ and $\left(x_{2}, y_{2}\right) \in B$. From this, choosing arbitrarily $x_{1}, x_{2} \in X$ and taking $y_{1}:=U\left(x_{1}\right), y_{2}:=L_{0}\left(x_{2}\right)$, we get

$$
L\left(x_{1}\right)-U\left(x_{1}\right) \leq u_{0} \leq L\left(x_{2}\right)-L_{0}\left(x_{2}\right),
$$

Pick any $z \in Z$. Since $U(0)=0$, from (25) used with $x_{1}=0, x_{2}=z$ and with $x_{1}=0, x_{2}=-z$, we obtain $L_{0}(z) \leq L(z)$, $-L_{0}(z)=L_{0}(-z) \leq L(-z)=-L(z)$, and hence $L(z)=L_{0}(z)$. Now, take any $x \in X$. From (25) used with $x_{1}=x$ and $x_{2}=0$ we get $L(x) \leq U(x)$. This ends the proof. 
The proofs of $(3.10) \Longrightarrow(3.11)$ and (3.11) $\Rightarrow(3.12)$ are given in [6, Theorem 4.1] and [6, Theorem 4.2], respectively.

We now prove (3.12) $\Rightarrow$ (3.13). Set $U:=L_{1}, V:=L_{2}$ and $x_{0}=0$. Note that $\left.L_{1}(Z) \cap \operatorname{int}\left(-X^{+}\right)\right)=\operatorname{int}\left(L_{1}(Z) \cap\left(-X^{+}\right)\right) \neq \emptyset$ (see also [22]). By (3.12) there is $L_{0} \in \mathcal{L}_{\text {inv }}(X, R)$ with $L_{0}\left(L_{1}(z)\right)+L_{2}(z) \geq 0$ for each $z \in Z$. Thus we get (3.13).

We now prove (3.13) $\Rightarrow(3.9)$. Let $L_{1}(z):=-z$ and $L_{2}(z):=L_{0}(z)$ for each $z \in Z$. It is readily seen that $L_{1}$ is linear and $G$ equivariant. From (9) we get $L_{0}(z) \geq 0$ for every $z \in Z \cap X^{+}$. By (3.13) we find a function $L \in \mathcal{L}_{+, \text {inv }}(X, R)$ with $L(-z)+$ $L_{0}(z)=-L(z)+L_{0}(z)=0$ for each $z \in Z$. Thus, (3.9) follows.

Now, let $\operatorname{int}\left(R^{+}\right) \neq \varnothing$ and let us show (3.9) $\Rightarrow$ (3.6). Let $U: D(U) \rightarrow R$ be a convex $G$-invariant function, with $D(U) G$ invariant and $U(0)=0$. Let $C:=$ epi $U:=\{(x, y) \in X \times R, y \geq U(x)\}$. Since $U$ is convex, $G$-subinvariant and $U(0)=0$, it is not difficult to see that $C$ is a $G$-invariant convex cone with vertex $(0,0)$. So, in $X \times R$, we consider the order generated by the cone $C$. Moreover, since $\operatorname{int}\left(R^{+}\right) \neq \varnothing$, by [22, Theorem 2] we get $\operatorname{int}($ epi $U) \neq \varnothing$ and (int (epi $\left.\left.U\right)\right) \cap(Z \times R) \neq \emptyset$. Define the action of $G$ on $X \times R$ by $g(x, y)=(g x, y),(x, y) \in X \times R$. Let $L_{0} \in \mathcal{L}_{\text {inv }}(Z, R)$ be such that $L_{0}(z) \leq U(z)$ for each $z \in D(U) \cap Z$. Define $L^{\prime}: Z \times R \rightarrow R$ by $L^{\prime}(z, y)=-L_{0}(z)+y,(z, y) \in Z \times R$. It is not difficult to check that $L^{\prime}(z, y) \geq 0$ whenever $(z, y) \in(Z \times R) \cap$ (epi $U)$. By (3.9) there is $L^{\prime \prime} \in \mathcal{L}_{\text {inv }}(X \times R, R)$ such that $L^{\prime \prime}(z, y)=L^{\prime}(z, y)=-L_{0}(z)+y$ for each $(z, y) \in Z \times R$ and $L^{\prime \prime}(x, y) \geq 0$ for every $(x, y) \in$ epi $U$. Thus, $L^{\prime \prime}(0, y)=y$ for any $y \in R$. Set $L(x):=-L^{\prime \prime}(x, 0), x \in X$. It is not difficult to check that $L \in \mathcal{L}_{\text {inv }}(X, R)$. We get $L(x)=y-L^{\prime \prime}(x, y)$ for each $(x, y) \in X \times R$, and hence, when $y=U(x)$ we obtain $L(x) \leq y=U(x)$. Moreover, for every $z \in Z$ and $y \in R$, we have $L(z)=y-L^{\prime \prime}(z, y)=y-L^{\prime}(z, y)=L_{0}(z)$. Thus, the assertion follows.

\section{APPLICATIONS}

Let $\mathcal{T}$ be a partially ordered set, for instance the "time set". In [5] (see also the related bibliography, for a historical overview), we saw that an event can be viewed as a triple $\left(E, t_{0}, T\right)$, in which $t_{0} \in \mathcal{T}$ is the present time instant, at which one has a suitable state of knowledge, $E$ is a logical proposition and $\emptyset \neq T \subset \mathcal{T}$ is a set about which we know that, for each $t \in T$, either $E$ is true at $t$ or $E$ is false at $t$. Note that, in general, it is not required that $t_{0} \leq \wedge T$, because it is possible to examine not only the future, but also the past, for example when one does not have sufficient informations about. Thus, it is advisable to consider as "events" some sets of the type $\Pi_{t \in \mathcal{T}} B_{t}$, where the $B_{t}$ 's are subsets of a fixed abstract nonempty set $\tilde{R}$ and $B_{t}=\tilde{R}$ whenever $t$ does not belong to $T$, where $T$ is a suitable subset of $\mathcal{T}$. It is advisable to examine all subsets of $B_{t}$, without requiring, for example, that the $B_{t}$ 's belong to fixed structures, like $\sigma$-algebras, and to construct finitely additive (not necessarily $\sigma$-additive) $R$-valued set functions. To this aim, we use the next theorem, which is a consequence of Theorem 3.1 (see also [7]).

Theorem 4.1. (Tarski theorem) Let $\Omega \neq \emptyset$ be any set, $\mathcal{P}(\Omega)$ be the family of all subsets of $\Omega, \mathcal{A}$ be an algebra of subsets of $G, G \subset \Omega^{\Omega}$ be an amenable semigroup of functions, such that $g^{-1}(A):=\{\omega \in \Omega: g(\omega) \in A\} \in \mathcal{A}$ for every $A \in \mathcal{A}$ and $g \in G$, and $\psi: \mathcal{A} \rightarrow R^{+}$be a finitely additive $G$-invariant measure. Then $\psi$ admits a finitely additive $G$-invariant extension $\tilde{\psi}: \mathcal{P}(\Omega) \rightarrow R^{+}$.

Proof. Let $X$ be the space of all bounded real-valued functions defined on $\Omega$ and $Z$ be the linear subspace of $X$ generated by all characteristic functions $\chi_{A}$, with $A \in \mathcal{A}$ (that is, $\chi_{A}(\omega)=1$ if $\omega \in A$ and $\chi_{A}(\omega)=0$ if $\left.\omega \notin A\right)$. For every $f \in X$ and $g \in G$, set $(g f)(\omega):=f(g(\omega))$. It is not difficult to check that the $g$ 's are linear monotone homomorphisms, with respect to the above defined action of $G$ on $X$. For each $f \in Z, f=\sum_{i=1}^{n} r_{i} \chi_{A_{i}}$, where $A_{i} \in \mathcal{A}, i=1,2, \ldots, n$, put $\phi(f)=\sum_{i=1}^{n} r_{i} \psi\left(A_{i}\right)$. It is not difficult to see that $\phi$ is well-defined (that is does not depend on the representation of $f$ ), monotone, linear and $G$ invariant. Let $\phi^{+}: X \rightarrow R, \phi^{-}: X \rightarrow R$ be defined by

$$
\begin{aligned}
& \phi^{+}(x)=\bigwedge\{\phi(z): x \leq z, z \in Z\}, \\
& \phi^{-}(x)=\bigvee\left\{\phi(z): x \geq z^{\prime}, z^{\prime} \in Z\right\} .
\end{aligned}
$$

Fix arbitrarily $x \in X$. By monotonicity of $\phi$, for every $z, z^{\prime} \in Z$ with $z^{\prime} \leq x \leq z$ we get $\phi\left(z^{\prime}\right) \leq \phi(z)$. Taking the supremum and the infimum as in (26), we obtain $\phi^{-}(x) \leq \phi^{+}(x)$. Moreover, it is not difficult to check that $\phi^{+}(z)=\phi^{-}(z)=\phi(z)$ for each $z \in Z$.

We now prove convexity of $\phi^{+}$. Fix arbitrarily $x_{1}, x_{2} \in X$ and $\lambda \in[0,1]$, and pick $z_{1}, z_{2} \in Z$ with $x_{i} \leq z_{i}, i=1,2$. Then $\lambda x_{1}+(1-\lambda) x_{2} \leq \lambda z_{1}+(1-\lambda) z_{2}$, and by linearity of $\phi$ it is $\phi^{+}\left(\lambda x_{1}+(1-\lambda) x_{2}\right) \leq \phi\left(\lambda z_{1}+(1-\lambda) z_{2}\right)=\lambda \phi\left(z_{1}\right)+(1-$ $\lambda) \phi\left(z_{2}\right)$. From arbitrariness of $z_{1}$ and $z_{2}$ we obtain $\phi^{+}\left(\lambda x_{1}+(1-\lambda) x_{2}\right) \leq \lambda \phi^{+}\left(x_{1}\right)+(1-\lambda) \phi^{+}\left(x_{2}\right)$, that is convexity of $\phi^{+}$. Analogously, it is possible to see that $-\phi^{-}$is convex.

Now we claim that $\phi^{+}$is $G$-subinvariant. Fix arbitrarily $x \in X$ and $g \in G$. For every $z \in Z$ with $x \leq z$, we get $g x \leq g z$ and hence $\phi^{+}(g x) \leq \phi(g z)=\phi(z)$. Taking the infimum, we obtain $\phi^{+}(g x) \leq \phi(x)$, getting the claim. Analogously, it is possible to see that $-\phi^{-}$is $G$-subinvariant.

By Theorem 3.1, there exist $\tilde{\phi} \in \mathcal{L}_{\text {inv }}(X, R)$ and $u_{0} \in R$ with 


$$
\phi^{-}(x) \leq \tilde{\phi}(x)-u_{0} \leq \phi^{+}(x)
$$

for every $x \in X$. Since $\phi^{+}(0)=\phi^{-}(0)=0$, from (27) used with $x=0$ we obtain $u_{0}=\tilde{\phi}(0)=0$. Hence, $\tilde{\phi}(z)=\phi(z)$ for any $z \in Z$. Setting $\tilde{\psi}(C):=\tilde{\phi}\left(\chi_{C}\right), C \subset \Omega$, we get that $\tilde{\psi}$ is the required $R^{+}$-valued extension.

We now give our application of Theorem 4.1 to events.

Example 4.2. Let $R$ be a Dedekind complete vector lattice, $(\tilde{R}, \mathcal{B})$ be a measurable space, where $\mathcal{B}$ is an algebra of subsets of $\tilde{R}, \mathcal{T}$ be any nonempty set, $\Omega:=\tilde{R}^{\mathcal{T}}$ and set $\mathcal{D}:=\{\{f \in \Omega$ : there exist $t \in \mathcal{T}, B \in \mathcal{B}: f(t) \in B\}$. Note that $\mathcal{D}$ is not an algebra, and that the algebra $\mathcal{A}(\mathcal{D})$ generated by $\mathcal{D}$ is the family of all finite (disjoint) unions of sets of the type

$$
E:=\left\{\bigcap_{i \in \Lambda}\left\{f \in \Omega: f\left(t_{i}\right) \in B_{i}\right\}: \Lambda \subset \mathcal{T} \text { is finite }\right\}
$$

(see also [5]). If $G \subset \mathcal{T}^{\mathcal{T}}$ is a semigroup of functions $\tau$ and $f \in \tilde{R}^{\mathcal{T}}$, then define the action of $G$ on $\tilde{R}^{\mathcal{T}}$ by $(\tau f)(t):=f(\tau(t))$. It is not difficult to see that $\mathcal{D}$ and $\mathcal{A}(\mathcal{D})$ are $G$-invariant.

Let $u \in R^{+}, u \neq 0$, and $P_{t}: \mathcal{B} \rightarrow R, t \in \mathcal{T}$, be a family of finitely additive $G$-invariant measures, such that $P_{t}(\varnothing)=0$ and $P_{t}(\tilde{R})=u$ for each $t \in \mathcal{T}$. We get that $\left\{P_{t}(B): t \in \mathcal{T}, B \in \mathcal{B}\right\} \subset V[u]:=\left\{x \in R\right.$ : there exists $c \in \mathbb{R}^{+}$such that $\left.-c u \leq x \leq c u\right\}$. By Dedekind completeness of $V[u]$ and by the Kakutani representation theorem, there are a compact Hausdorff topological space $\Xi$ and an isomorphism $\iota$ from $V[u]$ into $\mathcal{C}(\Xi):=\left\{f \in \mathbb{R}^{\Xi}: f\right.$ is continuous $\}$, which maps $u$ into the function $\mathbf{1}_{\Xi}$, defined on $\Xi$ and which assumes the constant value 1 (see also $\left.[23,44]\right)$. As the $P_{t}$ 's are equibounded, then they can be viewed as $V[u]$-valued set functions. Put $P(E):=\iota^{-1}\left(\iota\left(P_{t_{1}}\left(B_{1}\right)\right) \cdot \iota\left(P_{t_{2}}\left(B_{2}\right)\right) \cdot \ldots \cdot \iota\left(P_{t_{q}}\left(B_{q}\right)\right)\right)$, for every set $E$ defined as in (28). Note that, by construction, for such $E$ 's we get $0 \leq P(E) \leq u$. If $\tilde{E} \in \mathcal{A}(\mathcal{D}), \tilde{E}=\cup_{i=1}^{n} B_{i}$, where $B_{i}$ is as in (28), $i=1,2, \ldots, n$, and the $B_{i}$ 's are pairwise disjoint, then set $\tilde{P}(\tilde{E}):=\sum_{i=1}^{n} P\left(B_{i}\right)$. It is not difficult to check that $\tilde{P}$ is a finitely additive $G$-invariant $V[u]$-valued set function on $\mathcal{A}(\mathcal{D})$. Note that, if $E$ is as in (28), then

$$
P\left(\tau^{-1}(E)\right)=\iota^{-1}\left(\iota\left(P_{\tau\left(t_{1}\right)}\left(B_{1}\right)\right) \cdot \iota\left(P_{\tau\left(t_{2}\right)}\left(B_{2}\right)\right) \cdot \ldots \cdot \iota\left(P_{\tau\left(t_{q}\right)}\left(B_{q}\right)\right)\right) .
$$

By Theorem 4.1, $\tilde{P}$ admits a finitely additive $G$-invariant $V[u]$-valued extension $\hat{P}$, defined on the family $\mathcal{P}\left(\tilde{R}^{\mathcal{T}}\right)$ of all subsets of $\tilde{R}^{\mathcal{T}}$, and in particular on the sets of the type $\Pi_{t \in \mathcal{T}} B_{t}$, as $B_{t}$ varies in the whole of $\mathcal{P}(\tilde{R})$ for every $t \in \mathcal{T}$ and does not belong necessarily to $\mathcal{B}$.

Some examples are when $\mathcal{T}=\mathbb{R}, a>0$ and $G$ is the group generated by the translation $\tau_{a}(t):=t+a, t \in \mathbb{R}$, or when $\mathcal{T}=\mathbb{N}$ and $G$ is the group $\Sigma$ of all permutations keeping fixed all but finitely many numbers, which is amenable but not abelian (see also [38]). Thus, in case of invariance, some (possibly) unknown data concerning some elements $t \in \mathcal{T}$ can be "replaced" by some known informations associated with other elements $t^{\prime} \in \mathcal{T}$, when we study our "probabilities" $P_{t}$.

Some other applications associated with the group $\Sigma$, related to exchangeable processes, can be found, for instance, in [4] (see also $[28,39])$.

Remark 4.3. Observe that both Dedekind completeness and (right) amenability of $\boldsymbol{G}$ are not only sufficient, but also necessary conditions in order that the sandwich-type theorems hold (see for instance [43] and [7]).

\section{CONCLUSIONS}

We proved some versions of Hahn-Banach and sandwich-type theorem related to convex subinvariant operators, taking values in a partially ordered vector space $R$, in the setting of convexity, using a technique of construction of invariant finitely additive $R$-valued means, which allows to find invariant linear $R$-valued operators from linear operators which are not necessarily invariant, whose existence is guaranteed by the classical results. We deduced some Fenchel duality-type theorem, subdifferential formulas, Krein-type extension theorems, and showed that all these results are equivalent to separation, Farkas and Kuhn-Tucker-type minimization theorems. We used some similar classical results holding without invariance and a technique, by means to which it is possible to construct an invariant $R$-valued mean on all bounded $R$ valued functions defined on an amenable semigroup of homomorphisms and to get an invariant linear functional from a not necessarily invariant linear functional. We gave some applications to Tarski-type extension theorems for finitely additive invariant probability measures and to the study of families of "time-indexed" events, where the invariance allows to "replace" the study of the involved events connected with some periods of time on which one has not enough informations with corresponding events dealing with other periods of time about which one has a wider knowledge.

Several kinds of minimization problems, which have various applications in convex analysis, Calculus of Variations, subdifferential calculus, reconstruction of images, measure theory, probability and other branches of Mathematics, can be studied in this setting, by considering invariance or equivariance as particular constraints.

\section{ACKNOWLEDGMENTS}

(a) Our thanks to Prof. Alexander V. Bukhvalov for his useful suggestions.
$6168 \mid \mathrm{P}$ a g e
council for Innovative Research 
(b) This work is supported by University of Perugia and the Italian National Group of Mathematical Analysis, Probability and Applications (G.N.A.M.P.A.).

\section{REFERENCES}

[1] Aubert, G. and Kornprobst, P., Mathematical Problems in Image Processing - Partial Differential Equations and the Calculus of Variations, Second Edition, Springer, Berlin-Heidelberg-New York, 2006.

[2] Bergounioux, M., Introduction au traitement mathématique des images - méthodes déterministes, Springer, BerlinHeidelberg-New York, 2015.

[3] Boccuto, A., Riesz spaces, integration and sandwich theorems, Tatra Mt. Math. Publ. 3 (1993), 213-230.

[4] Boccuto, A., Continuous invariant vector-valued means and applications, Atti Sem. Mat. Fis. Univ. Modena 43 (1995), 229-244.

[5] Boccuto, A., On a new approach of event, coherence and extensions of set functions, Int. J. Math. 2 (4) (2016), Paper 1, 1-11.

http://www.ijrgroup.com/IJR-Group-pdf/International-Journal-for-Mathematics/International-Journal-for-Mathematics-April1.pdf

[6] Boccuto, A., Hahn-Banach, sandwich and separation theorems for invariant functionals with values in ordered vector spaces and applications to nonlinear vector programming, Academic J. Appl. Math. Sci. 2 (6) (2016), 45-50.

http://arpgweb.com/pdf-files/ajams2(6)45-50.pdf

[7] Boccuto, A. and Candeloro, D., Sandwich theorems, extension principles and amenability, Atti Sem. Mat. Fis. Univ. Modena 42 (1994), 257-271.

[8] Boccuto, A. and Dimitriou, X., Convergence theorems for lattice group-valued measures, Bentham Science Publ., U. A. E., 2015. ISBN 9781681080109

[9] Boccuto, A., Gerace, I., and Pucci, P., Convex approximation technique for interacting line elements deblurring: a new approach. J. Math. Imaging Vision 44 (2) (2012), 168-184.

[10] Boccuto, A. and Sambucini, A. R., The monotone integral with respect to Riesz space-valued capacities, Rend. Mat. (Roma) (1996), 491-524.

[11] Borwein, J. M. and Zhu, Q. J., A survey of subdifferential calculus with applications, Nonlinear Analysis 38 (1999), 687-773; Addendum to: "A survey of subdifferential calculus with applications", ibidem 49 (2002), 295 - 296.

[12] Borwein, J. M. and Vanderwerff, J. D., Convex Functions: Constructions, Characterizations and Counterexamples, Cambridge Univ. Press, Cambridge, 2010.

[13] Bredon, G. E., Introduction to compact transformation groups, Academic Press, Inc., New York, 1972.

[14] Buskes, G., The Hahn-Banach theorem surveyed, Dissertationes Math. 327 (1993), 1-35.

[15] Chojnacki, W., Sur un théorème de Day, un théorème de Mazur-Orlicz et une généralisation de quelques théorèmes de Silverman, Colloq. Math. 50 (1986), 257-262.

[16] Cignoli, R., D’Ottaviano, I. M. L., and Mundici, D., Algebraic Foundations of many-valued Reasoning, Kluwer Acad. Publ., Dordrecht, 2000.

[17] Cluni, F., Costarelli, D., Minotti, A. M., and Vinti, G., Applications of Sampling Kantorovich Operators to Thermographic Images for Seismic Engineering, J. Comput. Anal. Appl. 19 (4) (2015), pp. 602-617.

[18] Day, M. M., Amenable semigroups, III. J. Math. 1 (4) (1957), 509-544.

[19] Dinh, N. and Mo, T. H., Generalizations of the Hahn-Banach theorem revisited, Taiwanese J. Math. 19 (4) (2015), 1285-1304.

[20] Di Nola, A. and Leuştean, I., Łukasiewicz logic and Riesz spaces, Soft Computing 18 (12) (2014), $2349-2363$.

[21] Elster, K.-H. and Nehse, R., Konjugierte Operatoren und Subdifferentiale, Math. Operationsforsch. u. Statist. 6 (4) (1975), 641-657.

[22] Elster, K.-H. and Nehse, R., Necessary and sufficient conditions for the order-completeness of partially ordered vector spaces, Math. Nachr. 81 (1978), 301-311.

[23] Filter, W., Representations of Archimedean Riesz Spaces - A Survey, Rocky Mt. J. Math. (3), (1994), $771-851$.

[24] Fuchssteiner, B. and Lusky, W., Convex cones, North-Holland Publ. Co., Amsterdam, 1981.

[25] Gajda, Z., Sandwich theorems and amenable semigroups of transformations, Grazer Math. Ber. 316 (1992), $43 a ̂ €$ “58.

[26] Goodearl, K. R., Partially ordered abelian groups with interpolation, Amer. Math. Soc., Providence, Rhode Island, 1986.

[27] Gwinner, J., Results of Farkas type, Numer. Funct. Anal. Optim. 9 (1987), 471-520.

[28] Kallenberg, O., Probabilistic symmetries and invariance principles, Springer, New York, 2005.

[29] Köthe, G., Topological vector spaces I, Springer-Verlag, Berlin-Heidelberg-New York, 1969.

[30] Kuhn, H. and Tucker, A. W., Non-linear programming, in: Proceedings of the Second Berkeley Symposium on Mathematical Statistical Problems, University of California Press (1951), 481-492. 


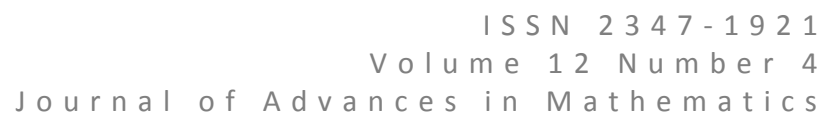

[31] Kusraev, A. G. and Kutateladze, S. S., Subdifferentials: Theory and applications, Kluwer Academic Publ., Dordrecht, 1995.

[32] Lipecki, Z., Compactness and extreme points of the set of quasi-measure extensions of a quasi-measure, Dissertationes Math. 493 (2013), 1-59.

[33] Mundici, D., Advanced Łukasiewicz calculus and MV-algebras, Springer, Berlin, 2011.

[34] Nehse, R., Some General Separation Theorems, Math. Nachr. 84 (1978), 319-327.

[35] Nehse, R., The Hahn-Banach property and equivalent conditions, Comm. Math. Univ. Carolinae 19 (1) (1978), 165177.

[36] Nehse, R., Separation of two sets in a product space, Math. Nachr. 97 (1980), 179-187.

[37] Nocedal, J. and Wright, S. J., Numerical Optimization, Second Edition, Springer, New York, 2006.

[38] Paterson, A. L. T., Amenability, Amer. Math. Soc., Providence, Rhode Island, 1988.

[39] Pratelli, L., La loi des grands nombres pour une suite éechangeable, Séminaire de Probabilités, XXIII, Lecture Notes Math. 1372, Springer, Berlin, 1989, 527-530.

[40] Rockafellar, R. T. and Wets, R. J.-B., Variational Analysis, Springer-Verlag, Berlin-Heidelberg-New York, 1998.

[41] Silverman, R., Invariant means and cones with vector interiors, Trans. Amer. Math. Soc. 88 (1) (1958), 75-79.

[42] Silverman, R. and Yen, T., Addendum to: "Invariant means and cones with vector interiors", Trans. Amer. Math. Soc. 88 (2) (1958), 327-330.

[43] To, T.-O., The equivalence of the least upper bound property and the Hahn-Banach extension property in ordered linear spaces, Proc. Amer. Math. Soc. 30 (2) (1971), 287-295.

[44] Wright, J. D. M., The measure extension problem for vector lattices, Ann. Inst. Fourier (Grenoble) 21 (1971), 65-85.

[45] Zowe, J., A duality theorem for a convex programming problem in order complete vector lattices, J. Math. Anal. Appl. 50 (1975), 273-287.

[46] Zowe, J., The saddle point theorem of Kuhn and Tucker in ordered vector spaces, J. Math. Anal. Appl. 57 (1977), 41. 55 .

[47] Zowe, J., Sandwich theorems for convex operators with values in an ordered vector space, J. Math. Anal. Appl. 66 (1978), 282-296.

\section{Author biography with Photo}

Antonio Boccuto was born in Catanzaro, Italy, on November 1964. He received the degree in Mathematics from the University of Perugia, Italy, in 1987, and the Ph.D. degree in Mathematical Analysis from the Mathematical Institute of the Slovak Academy of Sciences in Bratislava, Slovakia, in 2000. He received the habilitation in Mathematics to Associate Professor from the Comenius University in Bratislava in 2008. He has been a researcher in Mathematical Analysis at University of Perugia since the Academic Year 1991/1992. His research interests include Measure Theory and Integration, Real Analysis, Function Theory, Approximation Theory. He has published more than 120 papers on Mathematics in journals and conference proceedings and is a coauthor of two books on Measure and Integration Theory and Applications.

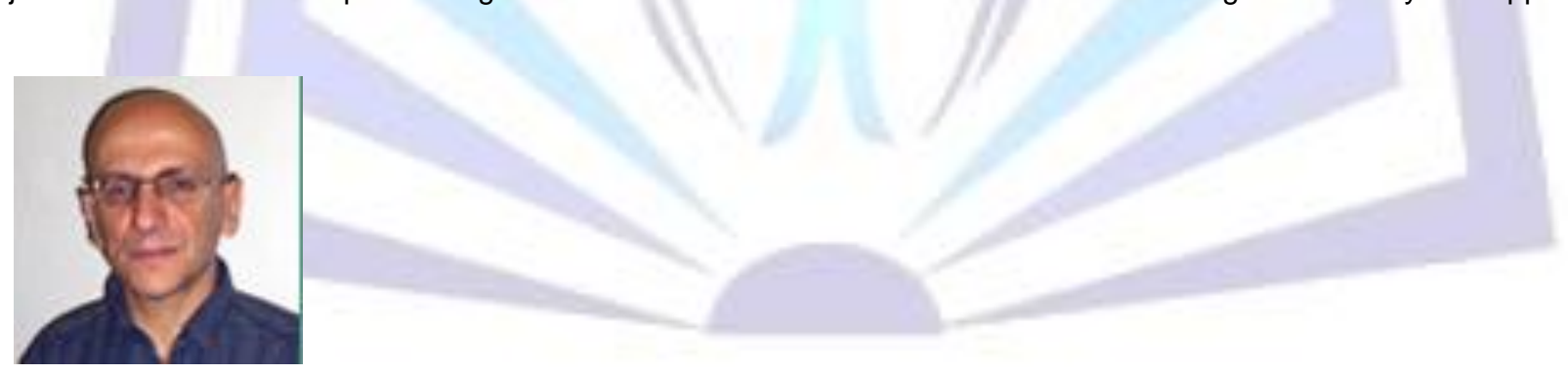

